\title{
GERVILLIA (GERVILLIA) SOLENOIDEA DEFRANCE, 1820 (BIVALVIA BAKEVELLIIDAE) DO NEO-APTIANO DE SERGIPE, BRASIL
}

\author{
MARIA HELENA HESSEL \\ Departamento de Biologia, UFS, Aracaju, Brasil. hesselmh@ufs.br
}

\begin{abstract}
RESUMO - No presente trabalho são descritos espécimes do biválvio baquevelídeo Gervillia (Gervillia) solenoidea, provenientes de duas localidades do município de Riachuelo, nordeste do Brasil: Mangueira 1 e Espírito Santo 11. Os exemplares foram coletados nos siltitos argilosos do membro Angico da formação Riachuelo, parte emersa da bacia de Sergipe. Citações equivocadas do gênero Gervillia em sedimentos brasileiros têm sido publicadas há mais de um século, mas estes espécimes são melhor relacionados ao gênero Aguileria White, 1887. A espécie epifaunal e epibissada Gervillia solenoidea possuia possivelmente um hábito de vida pendente em algas macroscópicas, vivendo em águas neríticas, subtropicais, claras e bem oxigenadas da plataforma continental interna formada pelas primeiras ingressões marinhas no nordeste brasileiro, quando do estabelecimento da livre circulação do Atlântico no Neo-Aptiano. O gênero e a espécie, até o momento não descritos nem ilustrados em sedimentos brasileiros, são associados ao amonóide Cheloniceras sp., sendo a ocorrência datada como neoaptiana.
\end{abstract}

Palavras-chave: Bivalvia, baquevelídeo, Eocretáceo, paleoecologia, bacia de Sergipe, Brasil.

\begin{abstract}
GERVILLIA (GERVILLIA) SOLENOIDEA DEFRANCE, 1820 (BIVALVIA BAKEVELLIIDAE) FROM THE APTIAN OF SERGIPE, BRAZIL. The bakevellid bivalve Gervillia (Gervillia) solenoidea is described from two localities from Riachuelo District, Northwest Brazil: Mangueira 1 and Espírito Santo 11. The specimens come from siltstones from Angico Member of Riachuelo Formation, onshore part of the Sergipe Basin. Mistake citations of the genus Gervillia in the Brazilian sediments have been published for more than a century, but these especimens are better related to the genus Aguileria White, 1887. The epifaunal epibyssate Gervillia solenoidea probably had a habit pendent to macroalgae, living in well-iluminated and well-oxygenated subtropical neritic waters of the internal continental platform. This environment was formed by the early marine ingressions on the Brazilian Northwest, when the Atlantic free circulation was established during the Late Aptian. The genus and the species, hitherto undescribed in Brazilian sediments are associated to the ammonite Cheloniceras sp., dated as Late Aptian.
\end{abstract}

Key words: Bivalvia, bakevellid, Early Cretaceous, paleoecology, Sergipe Basin, Brazil.

\section{INTRODUÇÃO}

O gênero Gervillia parece representar um grande número de formas de biválvios dos mares jurássicos e cretáceos, sempre com suas conchas finas e transversalmente muito alongadas. A revisão taxonômica efetuada por Muster (1995) inclui oito espécies, sem mencionar qualquer ocorrência sulamericana, ainda que já registrada no Peru (Jaworsky, 1915, 1925) e Argentina (Damborenea, 1987), ou africana: África do Sul (Rennie, 1936), Congo (Dartevelle \& Freneix, 1957), Moçambique (Silva, 1965) e Madagascar (Collignon, 1968). No presente trabalho, são descritas e ilustradas conchas de Gervillia (Gervillia) solenoidea provenientes da parte emersa da bacia de Sergipe, coletados pela autora em 1988, nos siltitos-argilosos eo-cretácicos do Membro Angico da Formação Riachuelo, no município de Riachuelo, nordeste do Brasil. Esta ocorrência sergipana é indubitavelmente neoaptiana por sua associação ao amonóide Cheloniceras sp.

Pela primeira vez no Brasil, o gênero Gervillia é descrito e ilustrado. Citações equivocadas do gênero em sedimentos brasileiros têm sido publicadas há mais de um século. White (1887a:54) descreveu quatro espécimes de um biválvio que denominou Gervillia dissita, ilustrando apenas um. Provinham de estratos cretácicos da região de Porto dos Barcos, no Estado de Sergipe, Brasil. No mesmo ano, o próprio White (1887b:37), relacionou esta espécie ao gênero Aguileria, por ele criado. Meio século depois, Maury (1937:98-100, est.11.67) descreveu e ilustrou Gervilleia regoi com base em exemplares da localidade de Posto da Garregoza, Sergipe. As conchas descritas são subquadradas, pouco oblíquas e muito espessas, características que não concordam com as feições morfológicas de Gervillia (ver adiante), sendo melhor referi- 


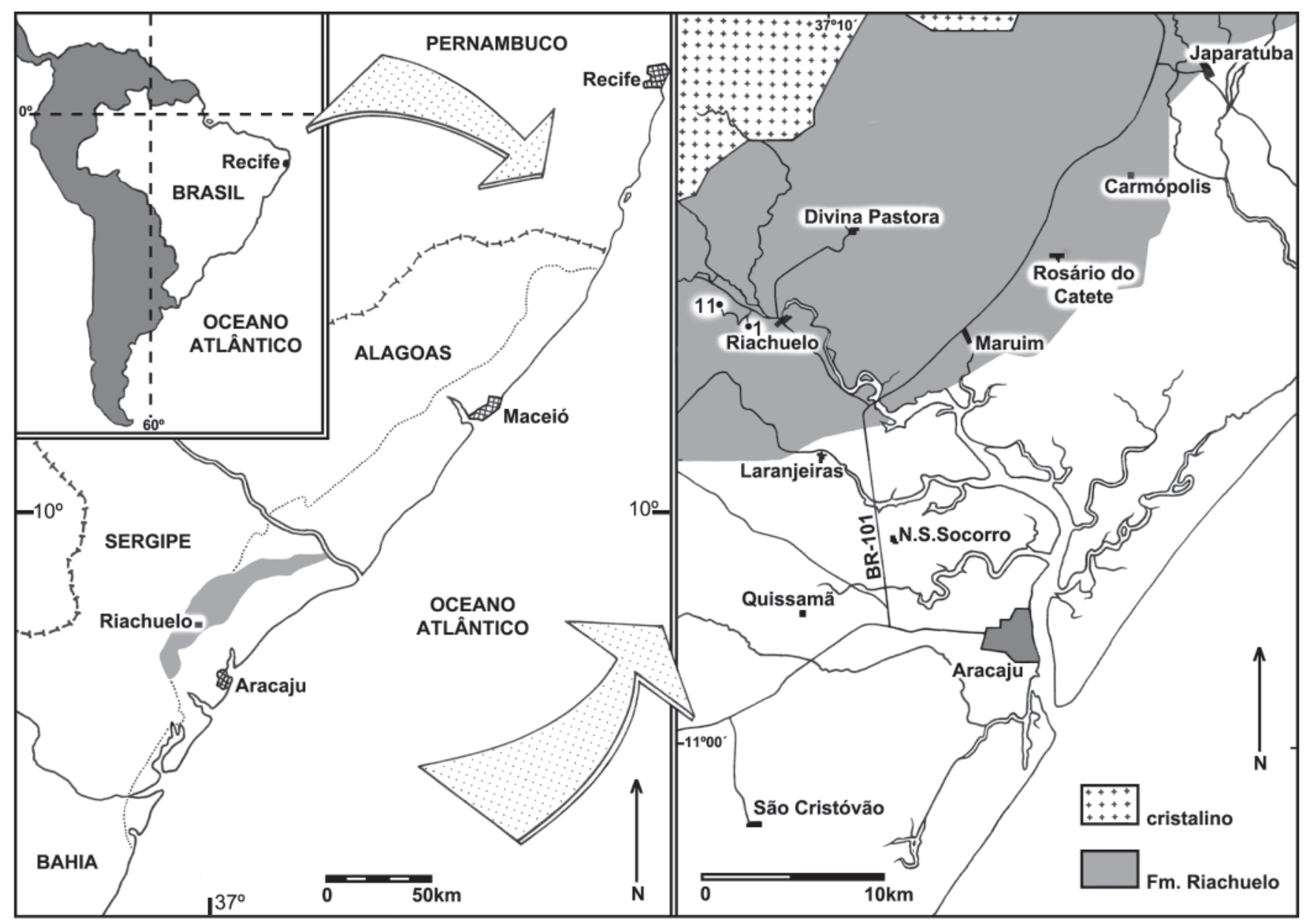

Figura 1. Localização geográfica da Formação Riachuelo (membro Angico) onde ocorre Gervillia (Gervillia) solenoidea no Estado de Sergipe, Brasil. 1 = Mangueira 1; 11 = Espírito Santo 11.

Figure 1. Location of Gervillia (Gervillia) solenoidea occurrences in Riachuelo Formation (Angico Member) from the Sergipe State, Brazil. 1 = Mangueira 1; 11 = Espírito Santo 11.

das ao gênero Aguileria, como já sublinhou Hessel \& Filizola (1989). Maury (1937:100, est.11.1) descreveu e figurou ainda os mesmos espécimes de G. dissita de White, mantendo a antiga designação, e indicando que dois deles provinham de Porto dos Barcos, um de Trapiche das Pedras Velho e um de Coqueiro, todas localidades de Sergipe. Branner (1915:348), Woodring (1926:844), Oliveira \& Leonardos (1943:566), Magalhães \& Mezzalira (1953:86, fig.92), Campos \& Campos (1976:184-187), Campos (1985:42) e Simone \& Mezzalira (1994:60) mencionam novamente as ocorrências descritas por White (1887a) e Maury (1937), sem questionamentos taxonômicos, ao contrário de Cassab et al. (1994:173) e Chagas et al. (2003:102), que reconhecem a sinonímia proposta por Hessel \& Filizola (1989). Kegel (1958:374) citou a presença de moldes de Gervillia nos arenitos da formação Beberibe, que datou como turoniana. Essa ocorrência foi novamente citada por Guimarães (1964:468), que observou vários exemplares dos gêneros Pteria e Gervillia no vale do rio Beberibe, a oeste de Recife (membro Beberibe da formação Itamaracá), em Pernambuco. Infelizmente, nenhum dos dois autores descreveu, ilustrou ou referiu o depósito destes fósseis, caso tenham sido coletados. Chagas et al. (2003:102) mencionam a presença de
Gervillia sp. na localidade de Coqueiro 1 em Sergipe, associada a amonóides do Eo-albiano (sem identificá-los), igualmente não descrevendo, ilustrando ou referindo o depósito destes exemplares de baquevelídeos.

A bacia de Sergipe, parte sul da bacia de Sergipe-Alagoas, e de onde provém as formas aqui descritas, apresenta uma das mais completas sequências sedimentares do Neomesozóico marinho brasileiro. Sua evolução tectônica iniciou-se com o distanciamento progressivo entre América do Sul e África, possivelmente durante o Neo-Aptiano, quando antigas falhas foram reativadas, formando na borda da bacia os conglomerados e arenitos sintectônicos intercalados a folhelhos, calcários e siltitos da formação Riachuelo (Neo-Aptiano/Cenomaniano). Em Sergipe, esta formação aflora numa faixa de cerca de $20 \mathrm{~km}$, subparalela ao litoral, indo desde o alto de Muribeca-Japoatão (no vale do rio Poxim, próximo a Pacatuba) ao norte, até pouco além do rio Vaza Barris (próximo a Itaporanga), ao sul (Figura 1). Está sobreposta aos calcilutitos e evaporitos da Formação Muribeca e sotoposta aos calcários de mar aberto da Formação Cotinguiba. Schaller (1970) definiu a Formação Riachuelo como uma sequência de carbonatos, folhelhos, siltitos e arenitos, representando uma sedimentação faciológica lateral. Esta variabi- 
lidade litológica gerou posteriormente a sub-divisão da formação em três membros estratigráficos (Feijó, 1994): Angico (de clásticos finos a grosseiros), Taquari (com folhelhos e calcilutitos intercalados) e Maruim (de calcarenitos e calcirruditos oolíticos, oncolíticos e/ou algálicos). O Membro Angico é constituído por arenitos conglomeráticos a finos, que gradam em direção ao centro da bacia para clásticos mais finos, folhelhos e siltitos.

\section{MATERIAL E MÉTODOS}

Os fósseis aqui estudados foram coletados na Formação Riachuelo, nas localidades fossilíferas de Mangueira 1 e Espírito Santo 11, no município de Riachuelo em Sergipe (Figura 1), onde ocorrem esparsamente. Ambas as localidades são a seguir descritas, conforme o sistema introduzido por Bengtson (1983). A sigla "Kra" significa Cretáceo, Formação Riachuelo, membro Angico (Bengtson, 1983):

MANGUEIRA 1: UTM 8812359N / 693832E. Mapa topográfico: SC.24-Z-B-IV Aracaju. Mapa geológico: SC.24-Z-B-IV-2 Riachuelo.

Seção em ambos os lados da rodovia, de cerca de $4 \mathrm{~m}$ de altura, SE ao poço da Petrobrás RO-357. As camadas são falhadas e mergulham cerca de $15^{\circ} \mathrm{NE}$. Altitude: $45-55 \mathrm{~m}$. Localidade descrita originalmente por Condé (1996).

Kra: siltito-argiloso maciço creme-claro.
ESPÍRITO SANTO 11: UTM 812350N/692050E. Mapa topográfico: SC.24-Z-B-IV Aracaju. Mapa geológico: SC.24-Z-BIV-2 Riachuelo.

Seção numa encosta dando para leste, junto ao poço da Petrobras RO-319. Altitude: cerca de $60 \mathrm{~m}$.

Kra: siltito-argiloso creme-claro muito friável.

A localidade de Espírito Santo 11, a noroeste da fazenda de mesmo nome, é uma localidade pouco fossilífera, sendo freqüentes apenas escamas de peixes. Lá foram coletados dois moldes externos de valvas esquerdas de Gervillia (Figura 5.5-6) e alguns fragmentos de pequenos amonóides indeterminados. Em Mangueira 1, próxima à fazenda Mata, foram coletados os restantes exemplares descritos neste estudo (Figura 5.1-4) e encontrados numerosos espinhos e fragmentos de equinóides regulares, escamas de peixes e conchas de pequenos turritelídeos; menos freqüentemente observados são tubos de serpulídeos, valvas de pequenos ostreídeos, conchas de Neithea, valvas inalteradas de Gervillia e diminutos biválvios e gastrópodos indeterminados; de ocorrência rara são conchas coloridas de neritídeos e restos de amonóides do gênero Cheloniceras (Peter Bengtson, comunicação verbal, 1989).

Na descrição que se segue, a terminologia morfológica adotada está de acordo com o explicitado na Figura 2. Todas as dimensões mencionadas neste trabalho estão em

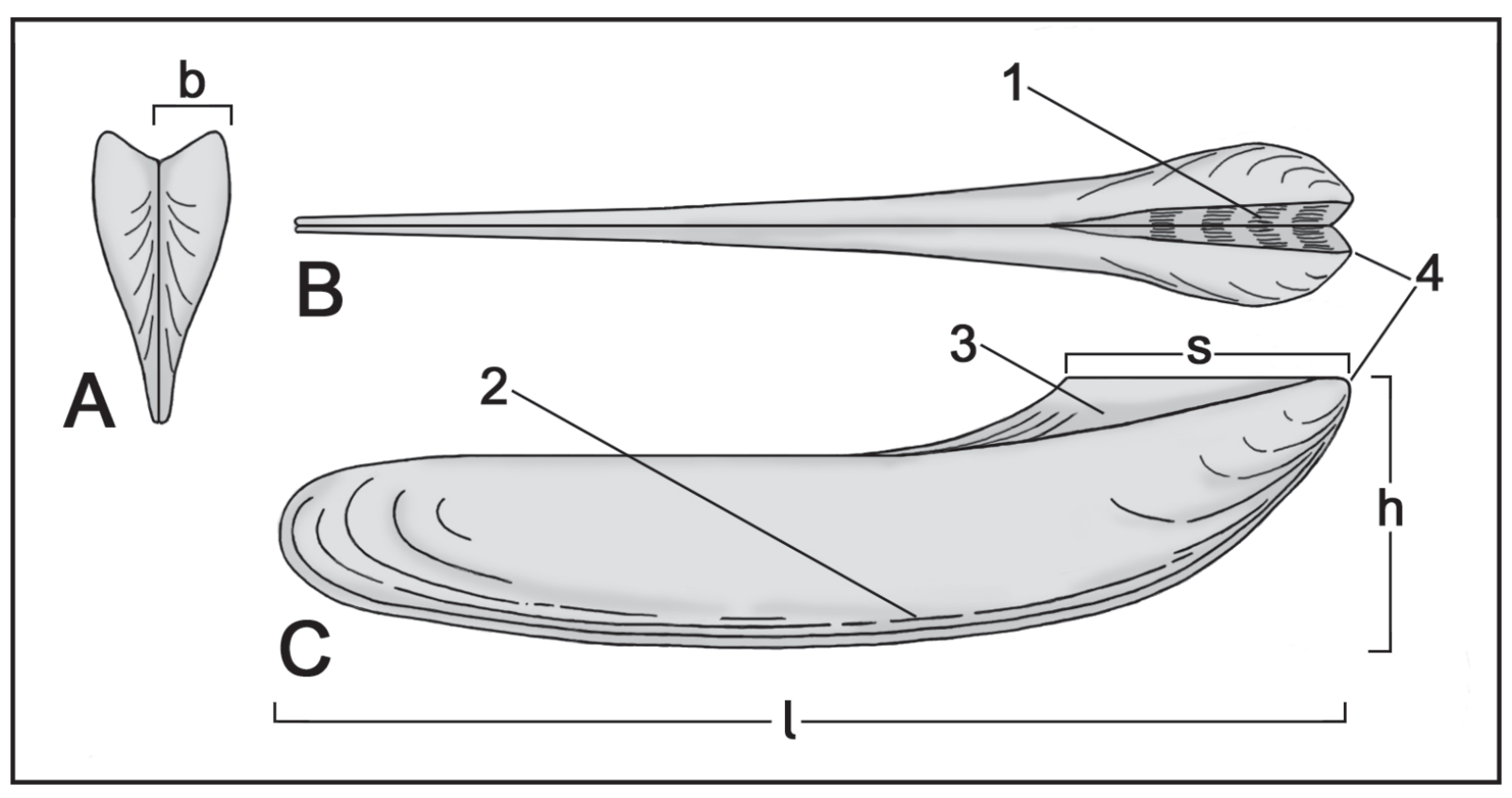

Figura 2. Terminologia morfológica e medidas de Gervillia solenoidea: A. Vista anterior; B. Vista dorsal; C. Vista lateral da valva direita. Medidas: s, dimensão máxima da linha cardinal; I, largura da valva (dimensão máxima entre as margens anterior e posterior, paralela à linha cardinal); $\mathbf{h}$, comprimento da valva (dimensão máxima perpendicular à I); b, convexidade máxima da valva; $\mathbf{1}$, área ligamentar; $\mathbf{2}$, linha de crescimento; 3, aurícula (posterior); 4, umbo.

Figure 2. Morphological terminology and measurements of Gervillia solenoidea: A. Anterior view; B. Dorsal view; C. Lateral view of right valve. Measurements: s, maximal dimension of hinge; I, greatest width (maximal dimension between anterior and posterior margins, parallel to the hinge); $\mathbf{h}$, greatest height (maximal perpendicular dimension to I); b, maximal convexity; 1, ligamental area; 2 , growth line; $\mathbf{3}$, auricle (posterior); $\mathbf{4}$, umbo. 
milímetros, e todas as conchas (não os moldes) foram preparadas de modo convencional, com broca vibradora. Os espécimes estão preservados como moldes externos ou com suas conchas, ainda que em geral com as porções posteriores perdidas. Estão depositados na Coleção de Paleoinvertebrados do Departamento de Paleontologia do Museu Nacional, Rio de Janeiro, sob os números de MN 6849-I a MN 6858 e MN 6887-I a MN 6889-I.

\section{TAXONOMIA}

\author{
Classe BIVALVIA Linné, 1758 pars \\ Subclasse PTERIOMORPHIA K.Beurlen, 1944 \\ Ordem PTERIOIDA Newell, 1965 \\ Sub-ordem PTERIINA Newell, 1965 \\ Superfamília PTERIACEA Gray, 1847 (1820) \\ Família BAKEVELLIIDAE King, 1850 \\ Gênero Gervillia Defrance, 1820
}

Diagnose. Concha muito longa e estreita, posteriormente alongada, com proporção diagonal máxima de seis vezes o tamanho da largura; umbo geralmente terminal; abertura bissal em geral bem visível (Muster, 1995:73).

Espécie-tipo. Gervillia solenoidea Defrance, 1820.

Gervillia (Gervillia) solenoidea Defrance, 1820 (Figura 5)

Gervillia solenoidea Defrance, 1820: 503.

Gervillia solenoides. Sowerby, 1826: 14, est. 510.1-4. Gervillia solenoides. Goldfuss, 1841: 124, est. 115.10a-b. Dalliconcha invaginata White, 1887b, est. 2.4-5.

Tabela 1. Medidas (em mm) dos espécimes de Gervillia (Gervillia) solenoidea da bacia de Sergipe, como apresentadas na Figura 2. Asteriscos indicam exemplares incompletos. O exemplar MN 6887-I é duvidosamente relacionado a uma valva esquerda.

Table 1. Measurements (in mm) of Gervillia (Gervillia) solenoidea specimens from Sergipe Basin, as presented in Figure 2. Asterisks indicate incomplete specimens. The specimen MN 6887-I is doubtly assigned to a left valve.

\begin{tabular}{c|cccc|cccc} 
& \multicolumn{4}{|c|}{ valva direita } & \multicolumn{4}{c}{ valva esquerda } \\
\cline { 2 - 9 } & $\mathbf{I}$ & $\mathbf{h}$ & $\mathbf{b}$ & $\mathbf{s}$ & $\mathbf{I}$ & $\mathbf{h}$ & $\mathbf{b}$ & $\mathbf{s}$ \\
\hline $\mathbf{6 8 4 9 - I}$ & $29,8^{*}$ & $14,0^{*}$ & 1,7 & $28,0^{*}$ & $29,8^{*}$ & $14,0^{*}$ & 1,7 & $28,0^{*}$ \\
$\mathbf{6 8 5 0 - I}$ & $36,8^{*}$ & $16,6^{*}$ & 3,2 & $18,5^{*}$ & $36,8^{*}$ & $16,6^{*}$ & 3,2 & $18,5^{*}$ \\
$\mathbf{6 8 5 1}-\mathrm{I}$ & $29,5^{*}$ & 18,0 & 3,2 & 28,0 & $29,5^{*}$ & 18,0 & 4,0 & 28,0 \\
$\mathbf{6 8 5 2 - I}$ & $40,5^{*}$ & 12,6 & 4,0 & 12,6 & $40,5^{*}$ & 12,0 & 4.0 & 12,0 \\
$\mathbf{6 8 5 3 - I}$ & $53,0^{*}$ & 26,0 & 6,4 & 38,5 & $57,0^{*}$ & 27,0 & 7,0 & 38,0 \\
$\mathbf{6 8 5 4 - I}$ & $27,0^{*}$ & 20,0 & 5,0 & 24,0 & $24,5^{*}$ & 20,0 & 5,0 & 23,6 \\
$\mathbf{6 8 5 5 - I}$ & $38,0^{*}$ & $16,0^{*}$ & $5,1^{*}$ & 32,0 & $38,0^{*}$ & $16,1^{*}$ & $4,1^{*}$ & 32,0 \\
$\mathbf{6 8 5 6 - I}$ & $44,9^{*}$ & 19,0 & 5,2 & 30,8 & - & - & - & - \\
$\mathbf{6 8 5 7 - I}$ & $21,5^{*}$ & $12,0^{*}$ & 5,5 & - & - & - & - & - \\
$\mathbf{6 8 5 8 - I}$ & $35,5^{*}$ & $14,0^{*}$ & 5,3 & $21,0^{*}$ & - & - & - & - \\
$\mathbf{6 8 8 7 - I}$ & - & - & - & - & $22,0^{*}$ & $11,8^{*}$ & $5,0^{*}$ & 21,0 \\
$\mathbf{6 8 8 8 - I}$ & - & - & - & - & 52,1 & 12,4 & 2,2 & 22,8 \\
$\mathbf{6 8 8 9 - I}$ & - & - & - & - & $39,0^{*}$ & 13,0 & 2,3 & $21,0^{*}$ \\
\hline
\end{tabular}

Dalliconcha ensiformis. White1887b, est. 2.6.

Gervilleia aff. Gervilliopsis ensiformis Frech, 1902, 616.

Gervillia forbesiana. Woods, 1905: 85, est.11:26-27, est. 12.1-5. Gervillia forbesiana. Yabe, Nagao \& Shimizu, 1926: 57, est. 12.36-37, est.14:8-9.

Gervillia forbesiana. Yabe, 1927, est. 5.5a-b.

Gervillia forbesiana. Nagao, 1934: 197, est. 24.8, est. 25.8-10. Gervillia (Gervillia) solenoidea. Muster, 1995: 78-79, est. 15.1-5.

Diagnose. Abertura bissal visível como um tubo na margem antero-superior, sem aurícula anterior; dentes anteriormente crenulados que, no decurso da ontogenia, ficam posteriormente mais próximos da porção superior da charneira (Muster, 1995:78).

Material. Treze exemplares, sendo sete exemplares bivalves MN 6849-I a MN 6855-I, três valvas direitas MN 6856-I a MN 6858-I e três valvas esquerdas MN 6887-I a MN 6889-I.

Descrição. A concha é de porte mediano (Tabela 1), bastante inequilateral e levemente inequivalva: a valva esquerda parece ser mais inflada ou larga do que a direita (Figura 5:1b, 2c), como é característico aos baquevelídeos (McGhee, 1978). É estreita e obliquamente alongada (quase paralela à linha cardinal), um pouco curva (ensiforme), relativamente fina, sendo mais espessa em sua porção anterior. A margem anterior é bastante encurvada, convexa, e a margem ventral é levemente curva, sub-retilínea. A margem posterior é muito arqueada. $\mathrm{O}$ umbo é terminal e prosógiro, não muito proeminente (Figura 5:5). A abertura bissal é assinalada por uma reentrância suave na porção ântero-superior (Figura 5.3). Na porção anterior, a concha é claramente biconvexa, tornando-se cada vez mais achatada à medida que se aproxima da margem posterior (Figura $5.1 b, 2 c$ ). A relação h/b, nos sete espécimes onde é possível constatá-la, é em média de 4,5, variando de 3,1 a 5,6 (Tabela 1). Em toda a superfície externa da concha é possível observar finas linhas de crescimento. A linha cardinal é longa e retilínea. A aurícula posterior é alongada, subtriangular, lisa, variando entre 12,2 e 38,5 mm (Tabela 1). É separada do restante da concha por um sulco não muito profundo (Figura 5:1a, 4 e 6). Este sulco forma, com a margem dorsal, um ângulo agudo de aproximadamente $8^{\circ}$. A área ligamentar é triangular baixa, posteriormente muito alongada, com quatro fossetas transversais, indicando um ligamento multivincular opistodético bastante desenvolvido (Figura 5.3). As fossetas ligamentares têm uma largura que varia de 2 a $3 \mathrm{~mm}$, separadas por espaços que medem de 1,5 a $2 \mathrm{~mm}$. Os dentes da charneira e a marca muscular não são observáveis em nenhum dos exemplares.

Discussão. Os exemplares de Sergipe ora estudados pertencem ao gênero Gervillia por mostrar uma concha bastante estreita e obliquamente alongada, com abertura bissal ântero-superior e umbo terminal, no que concordam com a diagnose de Muster (1995:73). Também mostram só uma aurícula (a posterior), relativamente longa, e área ligamentar com quatro fossetas, o 


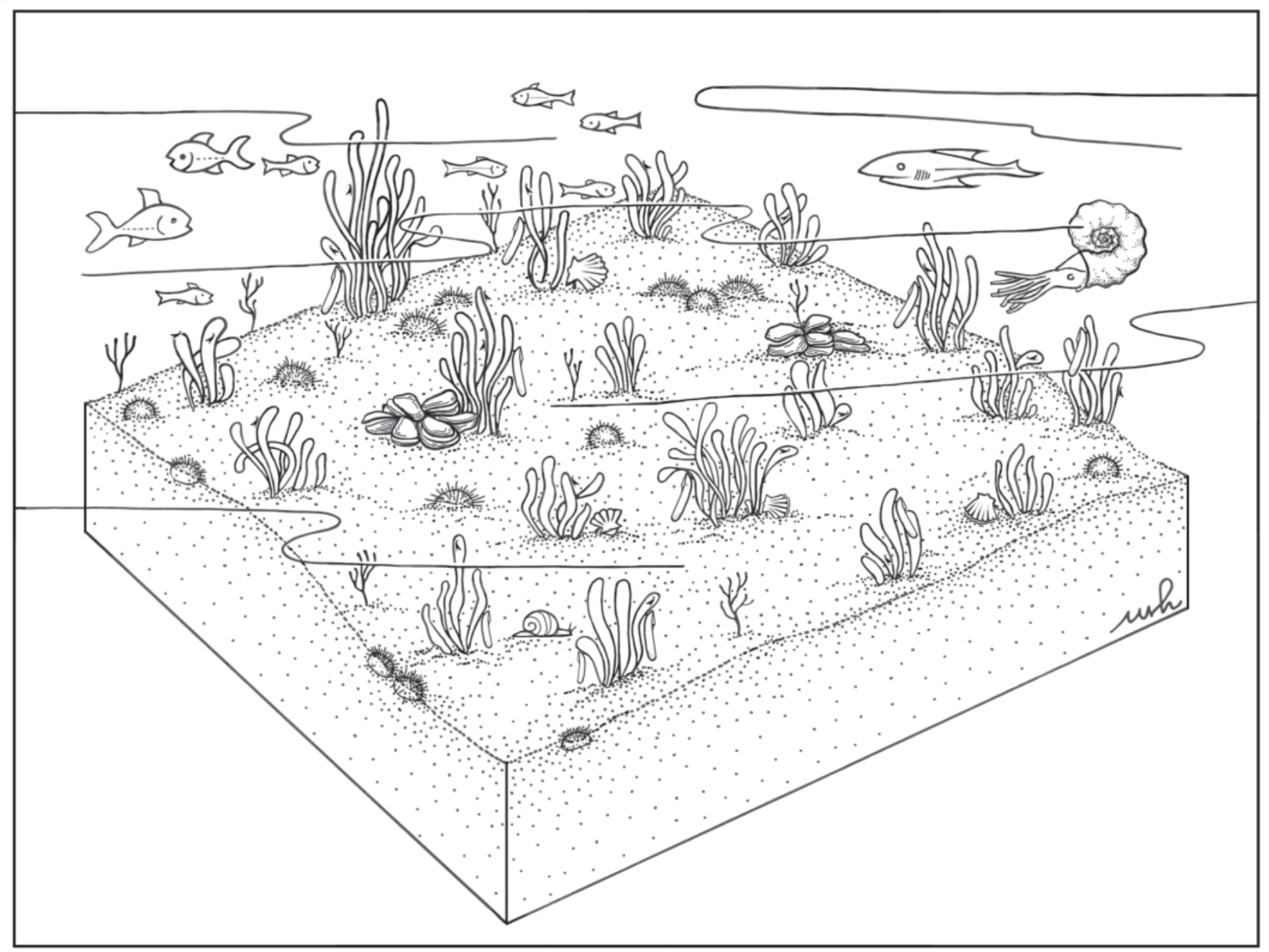

Figura 3. Reconstrução do ambiente no qual vivia Gervillia solenoidea do Neo-Aptiano de Sergipe: marinho raso com águas claras, subtropicais, bem oxigenadas e de boa circulação, com abundantes equinóides, peixes e possivelmente algas.

Figure 3. Paleoenvironmental reconstruction of Gervillia solenoidea in the Late Aptian of Sergipe: shallow and subtropical marine waters, well oxigenated, with echinoids, fishes and algae.

que está de acordo com as antigas diagnoses genéricas propostas por Dechaseaux (1952:277) e Cox (1969:N308). Discussões taxonômicas sobre o gênero Gervillia encontram-se com detalhe em Waagen (1907), Dietrich (1910), Gillet (1924), Cox (1946), Hayami (1975), Freneix (1965) e Damborenea (1987), não sendo aqui re-apresentadas. As características diagnósticas dos subgêneros, Gervillia Defrance, 1820 e Cultriopsis Cossmann, 1904, estão localizadas na charneira, não visível em nenhum dos exemplares de Sergipe. Entretanto, é possível relacioná-los ao subgênero Gervillia, por pertencer, a este, a espécie G. solenoidea. Por fim, os exemplares são referidos como G. solenoidea, já registrada nos sedimentos aptianos a maastrichtianos da Europa, África, Índia e Japão, por mostrarem muitas de suas feições morfológicas: concha ensiforme, oblíqua, estreita e alongada (comprimento estimado em cerca de quatro vezes maior do que a altura), com pequena e subtriangular aurícula posterior, quatro fossetas ligamentares e abertura bissal marcada por uma reentrância ântero-superior.

As formas aqui estudadas podem ser comparadas com outros gêneros de baquevelídeos de ocorrência brasileira.
Ao compará-las com o gênero Aguileria White, 1887b, já registrado no Albiano brasileiro (Hessel \& Filizola, 1989) e no Cenomaniano da América do Norte e Europa (Cox, 1969), observa-se que este gênero, a que devem ser referidas as descrições anteriores de Gervillia no Brasil, se caracteriza por ter uma espessa concha romboidal ou trapeziforme, com aurícula anterior, no que difere de G. (Gervillia) solenoidea.

Os exemplares sergipanos podem ser também comparados com outras espécies cretácicas do mesmo gênero e subgênero que apresentam um formato de concha semelhante. Assim, as formas aqui descritas diferem de G. (Gervillia) glaciana (Frech, 1902), conhecida de sedimentos neocretácicos da Europa (Muster, 1995), por possuir margem anterior arredondada, uma aurícula posterior subtriangular e área ligamentar bastante alongada. A espécie G. dentata Krauss, 1850 , encontrada na formação Witenhage (NeocomanianoAlbiano) da África do Sul (Rennie, 1936), mostra valvas mais convexas, espessas, pouco alongadas, e cinco grandes fossetas ligamentares, distinguindo-se deste modo de $G$. (Gervillia) solenoidea. 


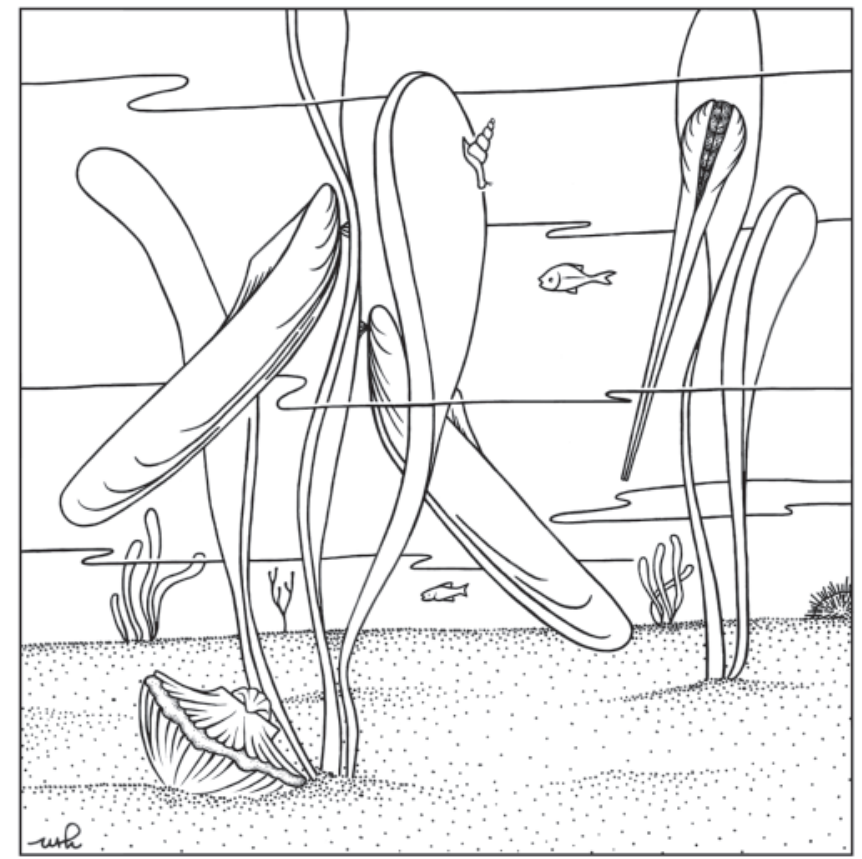

Figura 4. Reconstrução do modo de vida de Gervillia (Gervillia) solenoidea do Neo-Aptiano de Sergipe: uma forma epibissada pendente, presa a algas por bissus.

Figure 4. Reconstruction of the mode of life of Gervillia (Gervillia) solenoidea in the Late Aptian of Sergipe: a epibyssate pendant form, attached to the algae by a bissus.

Ocorrência. Localidades de Mangueira 1 e Espírito Santo 11, município de Riachuelo, na bacia de Sergipe, Estado de mesmo nome, Brasil, em siltitos-argilosos neo-aptianos do Membro Angico da Formação Riachuelo. Também ocorre em sedimentos aptianos a maastrichtianos da Alemanha, Áustria, Espanha, França, Inglaterra e Tchecoslováquia (Geinitz, 1842; Zittel, 1866; Guérander, 1867; Scupin, 1913; Wolansky, 1932; Dacqué, 1939; Muster, 1995), de Moçambique (Silva, 1965), Madagascar (Collignon, 1968), Índia (Dhondt, 1987) e Japão (Hayami, 1975).

\section{CONSIDERAÇÕES PALEOECOLÓGICAS}

Segundo Kauffman (1967), o gênero Gervillia corresponde a biválvios bissados epifaunais geralmente restritos a ambientes marinhos de alta energia da plataforma interna. Os abundantes restos de equinóides associados a Gervillia (Gervillia) solenoidea em Sergipe confirmam a origem francamente marinha desta ocorrência. Os ostreídeos e tubos calcários de vermes denunciam águas bem oxigenadas, com boa circulação, características de ambientes de alta energia. As conchas coloridas de neritídeos, além dos serpulídeos e ostreídeos, revelam águas rasas e claras da zona fótica, o que está de acordo com a atual interpretação de que os clásticos finos do Membro Angico foram depositados em águas rasas em frente a leques deltaicos (Cainelli et al., 1988). Assim, Gervillia solenoidea teria habitado a região marinha rasa da plataforma continental, possivelmente interna, entre os arenitos conglomeráticos mais próximos do continente e as argilas típicas da deposição de pró-delta da Formação Riachuelo.
Petri (1987) menciona que, durante o Albiano, a estreita plataforma carbonática existente na bacia de Sergipe-Alagoas era protegida por barreiras algais, estas sugerindo águas límpidas. É certo que algas, ainda que não observadas macroscopicamente nas duas localidades de onde provêm G. (G.) solenoidea, existiam no ambiente, como comprovam os estudos microfaciológicos de Bandeira (1978). Estas barreiras algais poderiam perfeitamente delimitar um ambiente de plataforma interna rasa, onde viveriam as formas de Gervillia, mesmo durante o Neo-Aptiano.

Dado o caráter cosmopolita do gênero, ocorrendo tanto em águas tropicais como temperadas (Dhondt, 1987), é difícil comentar sobre a temperatura das águas onde viveria Gervillia solenoidea em Sergipe. Entretanto, parece que não teria sido tropical, ainda que não se tenha evidências diretas para esta hipótese. Na fauna preservada, não há grandes indivíduos, típicos de mares quentes. A ausência de biválvios rudistas e de animais litoperfurantes nos mares cretáceos tem sido utilizada como indicador seguro de águas não tropicais (Kauffman, 1973). Efetivamente, não foram encontrados até o momento estes organismos em Mangueira 1 e Espírito Santo 11, ou em regiões circunvizinhas, sugerindo, para aquela época cretácica, águas sub-tropicais.

A macrofauna preservada junto com Gervillia solenoidea em Sergipe é bastante pobre (Figura 3). Há naturalmente a presença de peixes e pequenos amonóides natantes (Cheloniceras sp.), o que é esperado numa plataforma continental interna neoaptiana. Os seres bentônicos estão representados por abundantes equinóides regulares de pequeno porte e minúsculos gastrópodos turritelídeos. Ainda que os equinóides possivelmente vivessem semi-enterrados no substrato fino (siltito-argiloso) deste ambiente, os turritelídeos deveriam rastejar sobre algas. Há também pequenos ostreídeos cimentantes (que talvez formassem pequenos agrupamentos em torno da algum resto de concha ou fragmento de rocha), raros gastrópodos neritídeos (epifaunais vágeis; Hessel \& Carvalho, 1987), e uma pequena espécie de Neithea (Figura 4). A endofauna é bastante rara, o que poderia ser reflexo de um ambiente nerítico recentemente formado durante a ingressão marinha no nordeste brasileiro que estabeleceu a livre circulação do Atlântico durante o Neo-Aptiano (Feijó, 1996; Hessel \& Mello, 1997), sendo os invertebrados encontrados pertencentes a espécies pioneiras ou oportunistas, como deve ser o caso de G. (Gervillia) solenoidea.

Os baquevelídeos, família a qual pertence o gênero Gervillia, são sésseis, não enterrantes (McGhee, 1978). Em geral são monomiários e bissados (Muster, 1995) e o bissus tem uma importância significativa na estabilização do organismo (Stanley, 1972). Assim, G. (Gervillia) solenoidea viveria possivelmente presa por um bissus anterior às partes superiores de algas macroscópicas existentes na área (Bandeira, 1978; Figura 4). O modo epifaunal de vida de Gervillia é geralmente aceito por causa de suas valvas finas e achatadas, seu grande alongamento posterior e marca muscular única. O modo de vida de Gervillia dundriensis Cox, 1946 (sinônimo de G. lanceolata Goldfuss, 1841), encontrada nos Posidonia Shales (Eojurássico) da Alemanha, pendente em amonóides vivos, como foi sugerido por Seilacher (1982) e 
exemplificado como pseudoplâncton por Wignall \& Simms (1990), não pode ser aqui assumido, pois as conchas dos amonóides associados são muito pequenas, quase do mesmo tamanho do que as de Gervillia. Modo similar de vida é sugerido por Sellwood (1978) para formas de Gervillia do Eojurássico da Inglaterra, então pendentes em restos lenhosos de vegetais superiores flutuantes na água. Como não foi encontrado este tipo de resto fóssil nas localidades sergipenses aqui estudadas, parece improvável que os representantes de Gervillia solenoidea da formação Riachuelo adotassem este substrato para se desenvolverem.

Em sua fase juvenil, G. (Gervillia) solenoidea é bem mais inflada, demonstrando que evoluiu a partir de ancestrais habitantes de substratos duros. Entretanto, não é propriamente um habitante secundário de substratos moles, sensu Seilacher (1984), pois aparentemente habitava algas macroscópicas, um substrato flexível acima do fundo do mar. A valva direita, levemente menor e mais achatada, provavelmente ficava junto ao substrato (alga), fornecendo assim maior estabilidade ao organismo, como observou Stanley (1970) em formas de biválvios inequivalvos. A aurícula posterior alongada providenciaria maior estabilidade vertical à $G$. (Gervillia) solenoidea, como o leme de um barco, permitindo a concha não rolar e ter seu bissus retorcido. O ligamento multivincular opistodético é bastante desenvolvido provavelmente para contrabalançar a pressão da água que, mesmo de pouca profundidade, poderia forçar o fechamento de valvas tão delgadas como são as de Gervillia.

Gervillia solenoidea, como biválvio epifaunal epibissado, se alimentaria possivelmente de material em suspensão na água circundante, não necessitando de grandes e muito desenvolvidos sifões e pé, como sugerem suas valvas de bordos subparalelos quando fechados, sem formar sinus pedial ou sifonal. Assim, as diferentes espécies deste gênero hoje extinto foram provavelmente formas epibissadas pendentes, presas a substratos móveis (conchas de amonóides vivos ou restos lenhosos flutuantes, por exemplo) ou flexíveis (como algas macroscópicas).

\section{CONSIDERAÇÕES FINAIS}

A ocorrência do gênero Gervillia é temporalmente restrita ao Triássico, Jurássico e Cretáceo, sendo duvidosas referências mais antigas (Sanchez, 1985) e mais novas (Cox, 1969). Sanchez descreveu exemplares coletados no Neocarbonífero/Permiano da Venezuela, atribuindo com dúvidas ao gênero Gervillia. Pelas suas ilustrações (Sanchez, 1985, est. 3.12, 14-15), parece realmente tratar-se de outra forma alongada e pouco conhecida de biválvio.

Os fragmentos de Cheloniceras sp., encontrados na localidade Mangueira 1, associados às conchas de Gervillia solenoidea aqui descritas, datam estas camadas da Formação Riachuelo como neo-aptianas, de modo que o presente registro encontra-se perfeitamente dentro dos limites de ocorrência temporal do gênero e da espécie às quais as formas sergipanas são referidas.

Ocorrências de Gervillia em estratos cretácicos da
América do Sul são encontradas apenas em Domeyko (1851), que revelou a ocorrência de seus representantes no Eocretáceo do Chile, sem entretanto descrevê-los ou ilustrálos. Do México, Böse (1910) descreveu uma valva incompleta de Gervillia do grupo de G. solenoidea no eocenomaniano. De sedimentos peruanos (Jaworsky, 1915, 1925) e argentinos (Damborenea, 1987) são conhecidas só formas jurássicas, pertencentes ao sub-gênero Cultriopsis. No Brasil, ainda que o gênero tenha sido citado em Pernambuco (Kegel, 1958; Guimarães, 1964) e em Sergipe (Chagas et al., 2003), até o momento não se dispunha de um registro descritivo e ilustrado que comprovasse sua real presença em sedimentos brasileiros, sendo pioneiro o estudo aqui apresentado.

$\mathrm{Na}$ África, todas as informações referem-se a ocorrências bem mais recentes do que o presente registro em Sergipe. Da África do Sul, Rennie (1936) descreveu G. dentata Krauss, 1850, encontrada na formação Witenhage (NeocomanianoAlbiano). No Congo, Gervillia sp. foi reconhecida a partir de um exemplar mal preservado mencionado por Dartevelle \& Freneix (1957), proveniente de sedimentos cenomanoturonianos. De Moçambique, Silva (1965) listou a presença de Gervillia solenoidea em terrenos neocretácicos e, de Madagascar, Collignon (1968) citou a observação desta mesma espécie em sedimentos de diversas localidades maastrichtianas.

\section{CONCLUSÕES}

Com o presente estudo e análise de biválvios bakevelídeos coletados em sedimentos da bacia de Sergipe, é possível concluir que Gervillia (Gervillia) solenoidea Defrance, 1820 ocorre nos clásticos finos no Membro Angico da Formação Riachuelo no município de Riachuelo, Estado de Sergipe, contendo o presente trabalho a primeira descrição e ilustração do gênero em sedimentos brasileiros.

As formas de G. (Gervillia) solenoidea de Sergipe se caracterizam por uma concha de porte mediano, fina, ensiforme e levemente inequivalva, cuja porção anterior é biconvexa, e a posterior mais achatada. Possui linha cardinal longa e retilínea, aurícula posterior subtriangular, e área ligamentar triangular alongada com quatro fossetas transversais. Os espécimes de G. (Gervillia) solenoidea sugerem, pela morfologia de suas conchas, um hábito de vida epifaunal pendente, ficando presas pelo bissus a algas macroscópicas, constuindo-se em formas de biválvios suspensívoros habitantes secundários de substratos flexíveis.

O ambiente onde vivia G. (Gervillia) solenoidea devia ter sido marinho raso, de águas sub-tropicais claras, bem oxigenadas e de boa circulação, típico de plataforma continental interna da região durante o Neo-aptiano. A fauna associada sugere um ambiente nerítico recentemente formado durante as primeiras ingressões marinhas no nordeste brasileiro que levaram à livre circulação do Atlântico no Neo-aptiano, sendo as formas de Gervillia em Sergipe pioneiras ou oportunistas.

Gervillia (Gervillia) solenoidea encontra-se associada ao amonóide Cheloniceras sp., o que data sua ocorrência como neo-aptiana. 

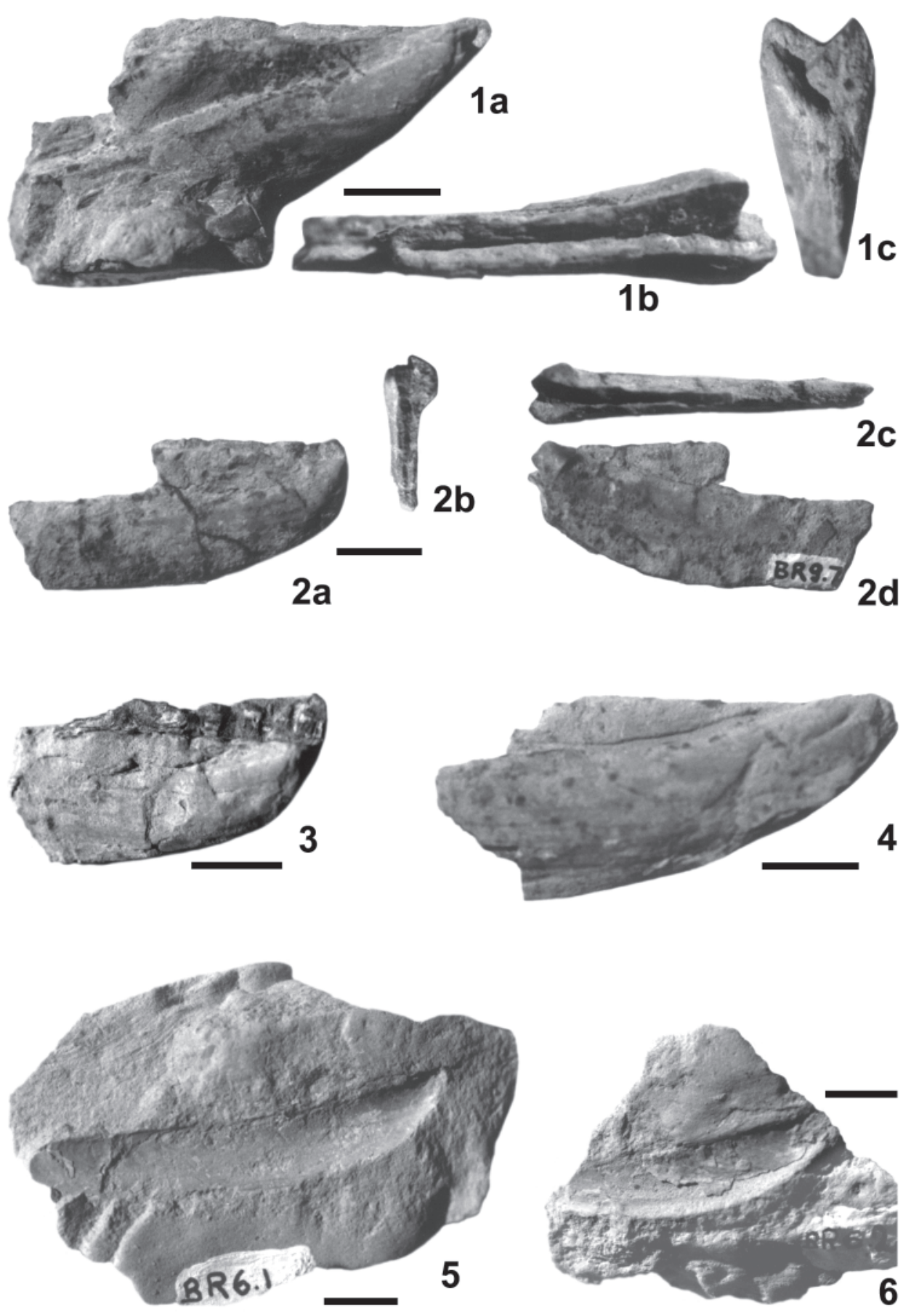

Figura 5. Gervillia (Gervillia) solenoidea Defrance, 1820 do Neo-Aptiano do município de Riachuelo, bacia de Sergipe, Brasil. 1. MN 6853I, procedente de Mangueira 1: a, vista externa da valva direita; b, vista dorsal de ambas as valvas; c, vista anterior de ambas as valvas. 2. MN 6852-I, procedente de Mangueira 1: a, vista externa da valva direita; b, vista anterior de ambas as valvas; c, vista dorsal de ambas as valvas; d, vista externa da valva esquerda. 3. MN 6855-I, procedente de Mangueira 1: vista interna da valva esquerda mostrando a área ligamentar. 4. MN 6856-I, procedente de Mangueira 1: vista externa da valva direita. 5. MN 6888-I, procedente de Espírito Santo 11: molde externo da valva esquerda. 6. Exemplar MN 6889-I, procedente de Espírito Santo 11: molde externo da valva esquerda (escalas =1 $\mathrm{cm}$ ). Figure 5. Gervillia (Gervillia) solenoidea Defrance, 1820, Late Aptian, Riachuelo Formation, Sergipe Basin, Brazil. 1. MN 6853-I from Mangueira 1: a, external view of right valve; $\mathbf{b}$, dorsal view of both valves; c, anterior view of both valves. 2. MN 6852-I from Mangueira 1: a, external view of right valve; $\mathbf{b}$, anterior view of both valves; $\mathbf{c}$, dorsal view of both valves; $\mathbf{d}$, external view of left valve. $\mathbf{3}$. Specimen MN 6855-I from Mangueira 1: internal view of left valve, showing the ligamental area. 4. Specimen MN 6856-I from Mangueira 1: external view of right valve. 5. Specimen MN 6888-I from Espírito Santo 11: external mould of the left valve. 6. Specimen MN 6889-I from Espírito Santo 11: external mould of the left valve (scale bars $=1 \mathrm{~cm})$. 


\section{AGRADECIMENTOS}

Nossos melhores agradecimentos a Susana Damborenea (Museo de Ciencias Naturales de La Plata) pela leitura crítica e diversas contribuições que muito enriqueceram o trabalho, a Peter Bengtson (Heidelberg Universität) pelas datações dos amonóides, a Ulf Gregor Baranow (UFPR) pela colaboração no trabalho de campo e a Marco Tulio Naves de Carvalho (UNB) pelo auxílio na medição dos exemplares e busca da extensa literatura.

\section{REFERÊNCIAS}

Bandeira Jr, A.N. 1978. Sedimentologia e microfácies calcárias das formações Riachuelo e Cotinguiba da bacia de Sergipe/Alagoas. Boletim Técnico da Petrobras, 21(1): 17-69.

Bengtson, P. 1983. The Cenomanian-Coniacian of the Sergipe Basin, Brazil. Fossils \& Strata, 12: 1-78.

Böse, E. 1910. Monografía geológica y paleontológica del Cerro de Muleros, Mexico, Instituto Geológico de Mexico, 193 p.

Branner, J.C. 1915. Geologia Elementar. Rio de Janeiro, Francisco Alves \& Cia, 350 p.

Cainelli, C.; Babinski, N.A.; Santos, R.C.R. \& Uesugui, N. 1988. Sedimentos albo-santonianos da bacia de Sergipe-Alagoas: ambientes de sedimentação e perspectivas petrolíferas. Revista Brasileira de Geociências, 17(2): 135-138.

Campos, D.R.B. 1985. Catálogo de fósseis-tipo e figurados das coleções paleontológicas do DNPM. 1. Invertebrados. Boletim do DNPM, Série Geologia, 26: 7-99.

Campos, D.R.B. \& Campos, D.A. 1976. Localidades fossilíferas da folha Aracaju-SC.24. In: C. Schobbenhaus $\mathrm{F}^{\mathrm{o}}$ (coord.) Carta geológica do Brasil ao milionésimo. Folha Aracaju (SC.24). Departamento Nacional da Produção Mineral, p. 167-204.

Cassab, R.C.T.; Santos, M.E.; Fernandes, A.C.; Brito, I.M.; Carvalho, I.S.; Coimbra, J.C.; Moura, J.A.; Duarte, L.; Freitas, L.C.; Hessel, M.H.; Carvalho, M.S.; Viviers, M.C. \& Uesugui, N. 1994. The Sergipe-Alagoas Basin. In: G. Beurlen; D.A.Campos \& M.C. Viviers (eds.) Stratigraphic Range of Cretaceous Mega- and Microfossils of Brazil, Universidade Federal do Rio de Janeiro, p. 161-231.

Chagas, R.R.D.; Zucon, M.H. \& Teodosio, C. 2003. Bivalves da família Bakevelliidae King, 1850, formação Riachuelo, Sergipe, Brasil. CONGRESSO BRASILEIRO DE PALEONTOLOGIA, $18^{\circ}$, 2003. Boletim de Resumos, Brasília, p. 102.

Collignon, M. 1968. Le Crétacé Supérieur da Madagascar. Memoir of the Geological Society of India, 2: 320-334.

Condé, V.C. 1996. Microgastrópodes do Eocretáceo de Sergipe, Brasil. Universidade Federal do Rio de Janeiro, Dissertação de Mestrado, 84 p.

Cossmann, M. 1904. Note sur 1'Infralias de la Vendée et des DeuxSévres. II. Pelécypodes. Bulletin de la Société Géologique de France, 4 série, 3(5): 497-537.

Cox, L.R. 1946. Undescribed Lamellibranch species from the English Inferior Oolite. Proceedings from the Malacological Society of London, 27(1): 22-32.

Cox, L.R. 1969. Family Bakevelliidae King 1850. In: R.C. Moore (ed.) Treatise on Invertebrate Paleontology, Part N(1), Boulder \& Lawrence, Geological Society of America \& University of Kansas Press, p. N306-N310.
Dacqué, E. 1939. Die Fauna der Regensburg-Kelheimer Oberkreide. Abhandlungen der Bayerischen Akademie der Wissenschaften, Neue Folge, 45: 1-218.

Damborenea, S.D. 1987. Early Jurassic Bivalvia of Argentina. Part 2: Superfamilies Pteriacea, Buchiacea and part of Pectinacea. Palaeontographica A, 199(4-6): 113-216.

Dartevelle, E. \& Freneix, S. 1957. Mollusques fossiles du Crétacé de la còte occidentale d'Africa, du Cameroun à l'Angola. 2. Lamellibranches. Annales du Musée Royale do Congo Belge, Sciences Géologiques, 20: 1-271.

Dechaseaux, C. 1952. Traité de Paléontologie. Paris, Masson et Cie, 289 p.

Defrance, M.J.L. 1820. Gervillie, Gervillia. Dictionaire des Sciences Naturelles, 18: 502-503.

Dhondt, A.V. 1987. Bivalves from the Hochmoos Formation (Gosau Group, Oberösterreich, Austria). Annalen des Naturhistorischen Museum zu Wien, 88(A): 41-101.

Dietrich, W.O. 1910. Ensigervilleia, eine neue Gervilliengruppe aus dem oberen weissen Jura von Schwaben. Centralblatt für Mineralogie, Geologie und Paläontologie, 8:235-242.

Domeyko, M.I. 1851. Mémoire sur les fossiles secondaires recueillis dans le Chili. Mémoire de la Société Géologique de France, $2^{\text {ème }}$ série, 4(11): 1-47.

Feijó, F.J. 1994. Bacias de Sergipe e Alagoas. Boletim de Geociências da Petrobras, 8(1): 149-161.

Feijó, F.J. 1996. O início da livre circulação das águas do Oceano Atlântico. Boletim de Geociências da Petrobras, 10(1/4):157164.

Frech, F. 1902. Über Gervilleia. Centralblatt für Mineralogie, Geologie und Paläontologie, 20: 609-620.

Freneix, S. 1965. Les bivalves du Jurassique Moyen et Supérieur du Sahara tunisien (Arcacea, Pteriacea, Pectinacea, Ostreacea, Mytilacea). Annales de Paléontologie, Invertebrés, 51(1): 51113.

Geinitz, H.B. 1842. Charakteristik der Schichten und Petrefacten des Sächsisch-böhmischen. Kreidegebirges, sowie der Versteinerungen von Kieslingswalda. Dresden \& Leipzig, Arnoldische Buchhandlung, $116 \mathrm{p}$.

Gillet, S. 1924. Études sur les Lamellibranches néocomienes. Memoires de la Société de France, Nouvelle Série, 3(1-2): 1339.

Goldfuss, G.A. 1841-1844. Petrefacta Germaniae, Teil III. Düsseldorf, Anstalt Arnz \& Co., 128 p.

Guérander, E. 1867. Album paléontologique du Département de la Sarthe. Le Mans, Imprimerie Beauvais et Valliénne, 25 p.

Guimarães, D. 1964. Geologia do Brasil. Rio de Janeiro, Departamento Nacional da Produção Mineral, 674 p.

Hayami, I. 1975. A systematic survey of the Mesozoic Bivalvia from Japan. Bulletin of the University Museum of the University of Tokyo, 10: 1-249.

Hessel, M.H. \& Carvalho, M.T.N. 1988. Padrão de coloração em Natica (Gastropoda) do Albiano Inferior de Sergipe. CONGRESSO BRASILEIRO DE PALEONTOLOGIA, $10^{\circ}$, 1987. Anais, Rio de Janeiro, 2: 457-469.

Hessel, M.H. \& Filizola Jr, N.P. 1989. Algumas espécies de Aguileria (Bivalvia) do Albiano Inferior de Sergipe. CONGRESSO BRASILEIRO DE PALEONTOLOGIA, $11^{\circ}$. Anais, Curitiba, SBG 1: 301-312.

Hessel, M.H. \& Mello, M.R. 1997. Caracterização das primeiras incursões marinhas na bacia de Campos por biomarcadores e biválvios. CONGRESSO BRASILEIRO DE GEOQUÍMICA, 6. Anais, Salvador, 2: 484-487.

Holzapfel, E. 1889. Die Mollusken der Aachener Kreide 2. Paläontographica A, 35: 139-268. 
Jaworsky, E. 1915. Beiträge zur Kenntnis des Jura in Südamerika, Teil II. Neues Jahrbuch für Mineralogie, Geologie und Paläontologie, 40: 364-456.

Jaworsky, E. 1925. Contribuición a la Paleontología del Jurásico Sudamericano. Publicación de la Direción General de Minería e Hidrología, Serie Geología, 4: 1-160.

Kauffman, E.G. 1967. Coloradoan macroinvertebrate assemblages, Central Western Interior, United States. In: E.G Kauffman \& H.C. Kent (eds), Paleo-environments of the Cretaceous Seaway: A Symposium. The University of Texas, p. 67-143.

Kauffman, E.G. 1973. Cretaceous Bivalvia. In: A. Hallam (ed.) Atlas of Paleobiogeography, Elsevier, p. 353-383.

Kegel, W. 1958. Um novo membro fossilífero da formação Itamaracá (Cretáceo Superior), Pernambuco. Anais da Academia Brasileira de Ciências, 29(3): 373-375.

Krauss, F. 1850. Über einige Petrefacten aus der unteren Kreide des Kaplandes. Verheinigen Königlichen Leopold-Carolus Akademie Naturforscher, 22(2): 441-464.

Magalhães, J. \& Mezzalira, S. 1953. Moluscos fósseis do Brasil. Rio de Janeiro, Departamento de Imprensa Nacional, 283 p.

Maury, C.J. 1937. O Cretaceo de Sergipe. Monografia do Serviço Geológico e Mineralógico do Brasil, 11:1-283.

Mcghee Jr, G.R. 1978. Analysis of the shell torsion phenomenon in the Bivalvia. Lethaia, 11: 315-329.

Muster, H. 1995. Taxonomie und Palaeobiogeographie der Bakevelliidae (Bivalvia). Beringeria, 14: 3-161.

Nagao, T. 1934. Cretaceous Mollusca from the Miyako District, Honshú, Japan. Journal of the Faculty of Sciences of Hokkaido Imperial University, 2(3): 177-277.

Oliveira, A.I. \& Leonardos, O.H. 1943. Geologia do Brasil, Rio de Janeiro, Imprensa Nacional, 813 p.

Petri, S. 1987. Cretaceous paleogeographic maps of Brazil. Palaeogeography, Palaeoclimatology, Palaeoecology, 59: 117-168.

Rennie, J.V.L. 1936. Lower Cretaceous Lamellibranchia from Northern Zululand. Annals of the South African Museum, 31: 277-391.

Sanchez, T.M. 1985. Étude paleoecologique du Paléozoique supérieur dans le Nord des Andes du Venezuela. Biostratigraphie du Paléozoique, 2: 1-165.

Schaller, H. 1970. Revisão estratigráfica da bacia de Sergipe/Alagoas. Boletim Técnico da Petrobras, 12(1): 1-65.

Scupin, H. 1913. Die Loewenberger Kreide und ihre Fauna. Paläontographica A, 6 (Suplement): 1-278.

Seilacher, A. 1982. Ammonite shells as habitats in the Posidonia Shales of Holzmaden: floats or benthic islands? Neues Jahrbuch für Geologie und Paläontologie, Monatshefte, 1982: 98-114.

Seilacher, A. 1984. Constructional morphology of bivalves: Evolutionary pathways in primary versus secondary softbottom dwellers. Palaeontology, 27(2): 207-237.
Sellwood, B.W. 1978: Jurassic. In: W.S. McKerrow (ed.) The ecology of fossils, The MIT Press, p. 204-279.

Silva, G.H. 1965. Contribuição para um catálogo dos lamelibrânquios fósseis do Cretácico de Moçambique. Revista de Estudos Gerais da Universidade de Moçambique, série 2, 2: 1-42.

Simone, L.R.L. \& Mezzalira, S. 1994. Fossil molluscs of Brazil. Boletim do Instituto Geológico, 11: 1-202.

Sowerby, J.C. 1826-1829. The mineral conchology of Great Britain VII. The Royal Society of London, London, $80 \mathrm{p}$.

Stanley, S.M. 1970. Relation of shell form to life habits of the Bivalvia (Mollusca). Memoir of the Geological Society of America, 125: 1-496.

Stanley, S.M. 1972. Functional morphology and evolution of byssaly attached bivalve mollusks. Journal of Paleontology, 46(2):165-212.

Waagen, I. 1907. Die Lamellibranchiaten der Pachycardientuffe der Seiser Alm nebst vergleichend paläntologischen und phylogenetischen Studien. Abhandlungen der Kaiserlichen Geologisch Reicjçhsen, 18:1-180.

White, C.A. 1887a. Contribuições à Paleontologia do Brasil. Archivos do Museu Nacional do Rio de Janeiro, 7:1-273.

White, C.A. 1887b. On new generic forms of Cretaceous Mollusca and their relation to other forms. Proceedings of the Academy of Natural Sciences of Philadelphia, 39: 32-37.

Wignall, P.B. \& Simms, M.J. 1990. Pseudoplancton. Palaeontology, 33(2): 359-377.

Wolansky, D. 1932. Die Cephalopoden und Lamellibranchiaten der Oberkreide Pommerns. Abhandlungen aus dem Geologisch-Paläontologischen Institut der Universität Greifswald, 9: 1-72.

Woodring, W.P. 1926. Fósseis terciáros do Brasil com descripção de nuovas formas cretaceas [rewiew]. Journal of Geology, 34: 843-846.

Woods, H. 1905. A monograph of the Cretaceous Lamellibranchia of England. Palaeontographical Society Monography, 59(2-3) [por 1904]: 57-96.

Yabe, H. 1927. Cretaceous stratigraphy of the Japanese islands. Science Report of the Tohoku Imperial University, 11(1): 27100.

Yabe, H.; Nagao, H. \& Shimizu, S. 1926. Cretaceous Mollusca from the Sanchu graben in the Kwanto mountainland, Japan. Science Report of the Tohoku Imperial University, 9(2): 33-76.

Zittel, K.A. 1866. Die Bivalven der Gosaugebilde in den nordaestlichen Alpen. Denkschriften der Kaiserlichen Akademie der Wissenschaften, 70: 77-198.

Received October, 2003; accepted January, 2004. 